\title{
Phlebologie
}

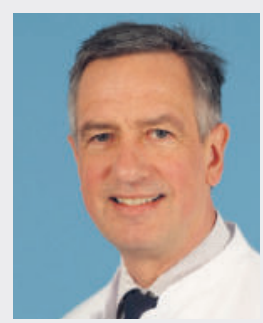

Prof. Dr. Achim Mumme, Bochum

\section{Operative Phlebologie: Varizenrezidive}

Aus der Sicht unserer Patienten steht die Vermeidung von Rezidiven an erster Stelle, wenn es darum geht, die Ansprüche an eine Varikosisbehandlung zu definieren. Dies zeigte eine Untersuchung von Shepherd et al. aus dem Jahr 2010 [2], bei der Patienten im Hinblick auf eine bevorstehende Krampfadernbehandlung befragt wurden. Es war das Varizenrezidiv, das den Befragten die größten Sorgen bereitete. Die Dauer der Rekonvaleszenz oder mögliche Beschwerden infolge der Behandlung waren dagegen von untergeordneter Bedeutung. Das Rezidivproblem war es wohl auch, das in den 80er Jahren in Deutschland zum Ende einer Ära der Varizenbehandlung führte. Die bis dahin dominierende Sklerosierungstherapie wurde von der Krampfadernoperation verdrängt, der invasiveren Methode, die aber im Vergleich weniger häufig rezidiviert.

Durch die Einführung der endovenösen Therapie ist abermals eine neue Ära angebrochen. Die neue Therapieoption konnte sich mit dem Versprechen ausbreiten, das Gleiche leisten zu können wie die Operation, nur eben schonender. Inzwischen sind die endovenösen Verfahren zumindest im angloamerikanischen Raum eindeutig zur Nummer 1 in der Behandlung der Stammveneninsuffizienz avanciert. Allerdings weiß bis heute niemand, wie deren Rezidivraten langfristig aussehen werden [1] und so ist auch über die endovenöse Therapie das letzte Wort noch nicht gesprochen worden. Möglicherweise könnte nämlich der neuen Therapieoption der sogenannte „endovenöse Crossenstumpf“ zum Verhängnis werden, der postinterventionell nahezu regelhaft verbleibt.

Mit dem Problem des Crossenstumpfes und daraus resultierender Crossenrezidive setzt sich die Arbeitsgruppe Mumme, Mühlberger, Sidhwa und Hummel mit dem Beitrag „Alarmierend hohe Rate saphenofemoraler Rezidive nach endovenöser Lasertherapie“ auseinander. Die im Vergleich zur Operation zu beobachtende hohe Rate duplexsonographisch detektierbarer Crossenrefluxe gibt Anlass zur Besorgnis. War es ein Fehler, bei der Einführung der endovenösen Verfahren das Prinzip der Crossektomie unberücksichtigt zu lassen?

Die Bedeutung der Crossektomie für den langfristigen Behandlungserfolg wird auch in der Übersichtsarbeit von Didi Stenger und Michael Hartmann thematisiert. Mit ihrem Beitrag „Die Chirurgie der Vena saphena parva - endoluminal oder operativ?“ geben zwei phlebologische Urgewächse ihre Erfahrungen weiter. Interessant sind hier nicht nur die wissenschaftlichen Aspekte, sondern auch die persönlichen Einschätzungen der Autoren im Hinblick auf die operativen und interventionellen Entwicklungen bei der Therapie der Parva-Insuffizienz.

Die Arbeitsgruppe Frings, Brümmer, Prinz, Glowacki und Rass beschäftigt sich schon seit vielen Jahren mit den Möglichkeiten zur Vermeidung von Rezidiven und hat zu diesem Thema bereits eine große Zahl von Publikationen und Kongressbeiträgen beigesteuert. Der Beitrag „Möglichkeiten zur Rezidivprophylaxe in der Varizenchirurgie“ gibt uns einen hervorragenden Überblick darüber, was wir tun können, um Varizenrezidive zu vermeiden. 
Das Thema Varizenrezidive wird abgerundet durch die kritische Analyse von Gerontopoulou und Rass „Therapie des inguinalen Rezidivs: Ist die offene Re-Crossektomie noch zeitgemäß?“. Ein wichtiger Beitrag in einer Zeit, in der die technisch anspruchsvolle Operation vor allem im angloamerikanischen Raum infrage gestellt wird.

Mit dem Leitthema „Operative Phlebologie: Varizenrezidive“ wird ein zentraler Aspekt der Phlebologie angesprochen, der nicht nur für Operateure relevant ist. Schließlich ist die Vermeidung von Rezidiven ein zentrales Ziel bei allen Therapieoptionen der Varikosis.

Bei der Lektüre dieser Ausgabe wünsche ich Ihnen Anregungen und viel Vergnügen Ihr

Prof. Dr. Achim Mumme, Bochum

\section{Literatur}

[1] Bosanquet DC, Twine CP. The Endovenous Literature: A Perfect Storm of Limited Effectiveness Data, Rapid Technological Evolution and Potential Conflict of Interest. Eur J Vasc Endovasc Surg 2017;54:771

[2] Shepherd AC, Gohel MS, Lim CS et al. The treatment of varicose veins: an investigation of patient preferences and expectations. Phlebology 2010;25:54-65 